\title{
Neutral Zone Technique for Rehabilitation of Resorbed Mandibular Ridge
}

\author{
${ }^{1}$ Avneet Kaur, ${ }^{2}$ Narendra Padiyar, ${ }^{3}$ Pragati Kaurani, ${ }^{4}$ Sudhir Meena, ${ }^{5}$ Devendra Pal Singh Chhonkar, ${ }^{6}$ Aditi Sarawgi
}

\begin{abstract}
The neutral zone technique is an alternative approach for the construction of the complete dentures. It is most effective for cases where there is a highly atrophic ridge. The technique aims to construct a denture, i.e., shaped by muscle function and is in harmony with the surrounding oral structures. This clinical report describes the fabrication of complete dentures for a patient with poorly formed mandibular ridges.
\end{abstract}

Keywords: Denture stability, Neutral zone, Resorbed ridge.

How to cite this article: Kaur A, Padiyar N, Kaurani P, Meena S, Chhonkar DPS, Sarawgi A. Neutral Zone Technique for Rehabilitation of Resorbed Mandibular Ridge. J Mahatma Gandhi Univ Med Sci Tech 2016;1(1):35-38.

\section{INTRODUCTION}

To increase the stability and retention of mandibular dentures, previous reports ${ }^{1,2}$ have recommended that posterior denture teeth be positioned directly over the crest of the edentulous ridge. This relationship of the teeth and alveolar ridge is important for the stability of the denture in function. ${ }^{1,2}$ Although this tooth position may be desirable when adequate alveolar ridge height is available, such a position may not be suitable for severely resorbed ridges because of a lateral shift in the orientation of the ridge crest. This horizontal discrepancy between original tooth position and resorbed ridge crest, coupled with an increased vertical distance between occlusal table and tissue support, can lead to denture instability. Successful denture treatment in situations like this can become increasingly dependent on the position of the denture teeth and the external contours of the dentures. It is for these patients that the neutral zone concept ${ }^{3-7}$ becomes increasingly significant. The lower denture commonly presents the most difficulties with pain and looseness being the most common complaint. ${ }^{8}$ This is because the mandible atrophies at a greater rate than themaxilla and has less residual ridge for retention and

\footnotetext{
${ }^{1}$ Postgraduate Student, ${ }^{2}$ Principal and Head, ${ }^{3}$ Professor, ${ }^{4}$ Reader ${ }^{5,6}$ Senior Lecturer

${ }^{1-6}$ Department of Prosthodontics and Crown and Bridges Mahatma Gandhi Dental College, Jaipur, Rajasthan, India
}

Corresponding Author: Avneet Kaur, 178, Ram Gali No.2 Raja Park, Jaipur, Rajasthan, India, Phone: +91-9587000888 e-mail: dravneet27@gmail.com support. ${ }^{9}$ The neutral zone technique is most effective for patients who have had numerous unstable, unretentive lower complete dentures. These patients usually have a highly atrophic mandible and there has been difficulty in positioning the teeth to produce a stable denture. ${ }^{10}$ Fish, in 1931, first described the influence of the polished surfaces on retention and stability. ${ }^{11} \mathrm{He}$ also described how dentures should be constructed in the 'dead space', which later came to be known as the neutral zone. ${ }^{3}$

The neutral zone has been defined as the potential space between the lips and cheeks on one side and the tongue on the other; that area or position where the forces between the tongue and cheeks or lips are equal. ${ }^{12}$ Two clinical studies have described the advantages of using the neutral zone technique. Cinefluorographic studies by Sheppard ${ }^{13}$ have shown that the muscles reseat the complete denture during function. In another clinical study, Fahmy and Kharat ${ }^{4}$ evaluated patients' chewing efficiency and satisfaction for complete dentures made with either a conventional or neutral zone technique. Patients had a better chewing efficiency when wearing conventional dentures, however, patients could not detect any difference in masticatory performance between their conventionally fabricated dentures and neutral zone dentures. Patients did report greater comfort and improved speech clarity with the dentures fabricated using the neutral zone technique compared with their conventional prepared dentures. These studies suggest that the neutral zone strategy for denture fabrication may be helpful in certain edentulous situations.

The purpose of this article is to present the use of the neutral zone technique for the fabrication of successful and stable maxillary and mandibular complete denture with severely resorbed ridges.

\section{CASE REPORT}

A 73-year-old male patient reported to the Department of Prosthodontics at Mahatma Gandhi Dental College, Jaipur, with a chief complaint of old unstable maxillary and mandibular complete denture. The patient was advised for the fabrication of complete denture with neutral zone technique as other treatment options like implant-supported complete dentures was not feasible due to the age and cost factors (Fig. 1). 


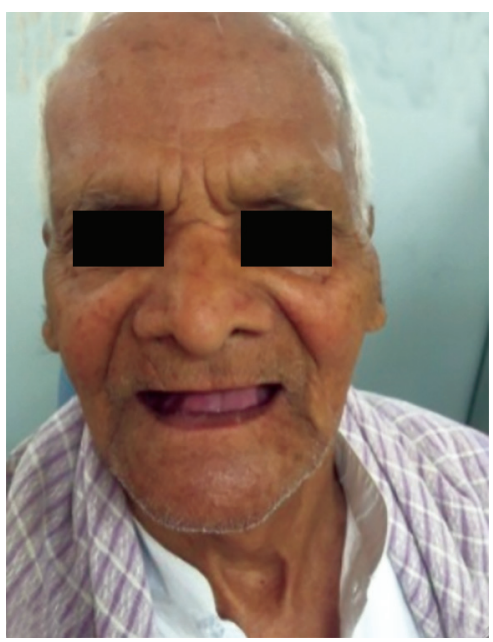

Fig. 1: Preoperative view

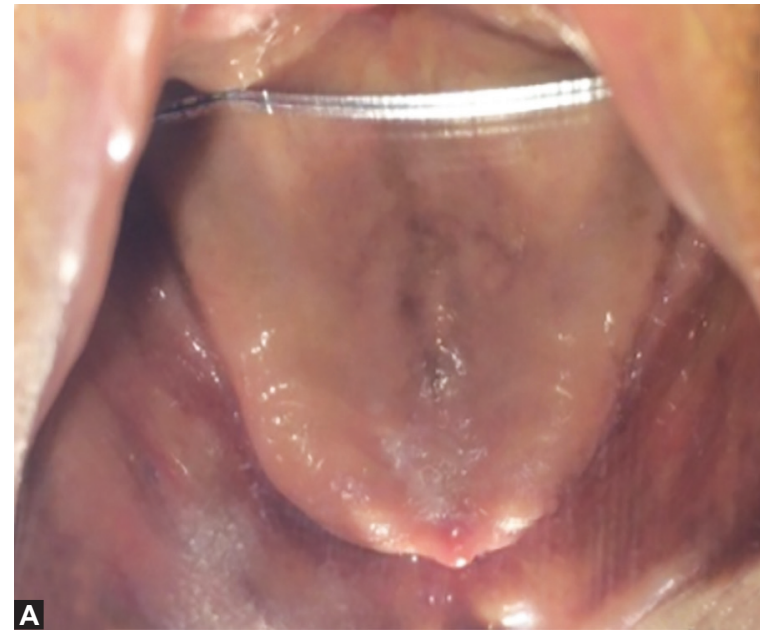

The lower ridge was highly resorbed whereas maxillary ridge was flabby in anterior region and slightly resorbed posteriorly on the both sides (Figs 2A and B).

- The primary impression was made using alginate impression material with a perforated edentulous stock tray.

- Closely fitting custom tray was fabricated and the border molding was performed with low fusing impression compound (green stick) to represent muscle activity, recording functional depth and width of the sulcus.

- The final wash impressions were made with poly vinyl siloxane light body material (Fig. 3).

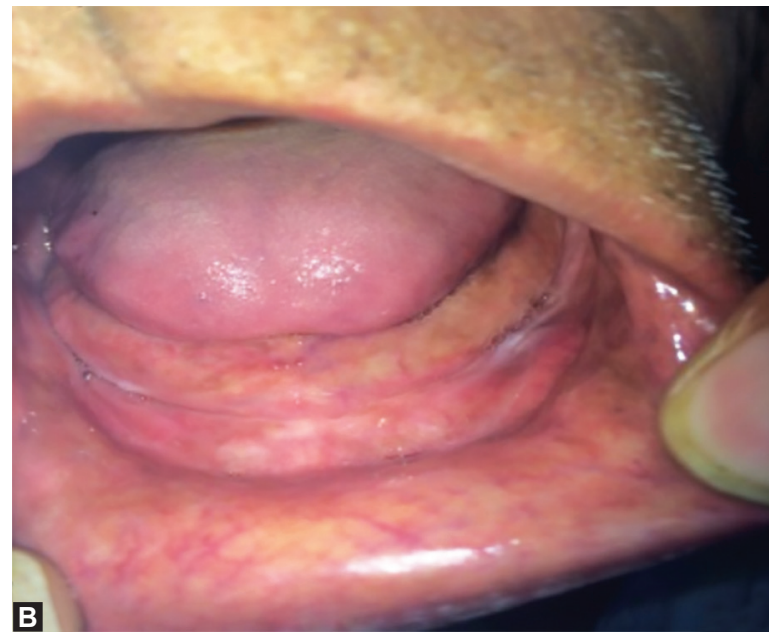

Figs 2A and B: Resorbed maxillary and mandibular ridges

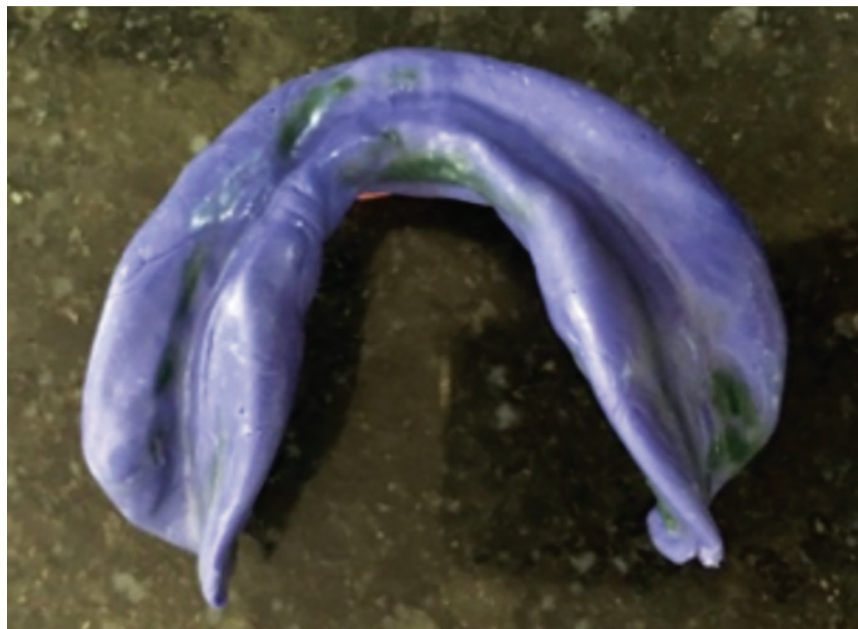

Fig. 3: Mandibular wash impressions

\section{Procedure}

A detailed examination was completed and his previous dentures were evaluated for retention and stability. The old denture was found to be unstable and was not retentive. Also, the old denture could not be relined.
- Impressions were evaluated for accuracy and master cast was poured with dental stone. Both the casts were then duplicated.

- The wax record rims were then constructed and assessed for extension, comfort, and stability.

- Jaw relation was carried out conventionally to record vertical and centric relation and the cast was articulated on mean value articulator.

- Then the other set of record rims were fabricated on the new denture bases on duplicate master cast. These rims were made up of type I impression compound.

- First the mandibular rim was adjusted at the same vertical height with maxillary rim maintaining the vertical stop.

- Before recording the neutral zone the patient was made to sit in a comfortable, upright position with the head unsupported.

- The mandibular compound rim was then inserted into patient's mouth and he was asked to perform a series of actions designed to simulate the physiological functioning, such as asking the patient to smile, grin, 

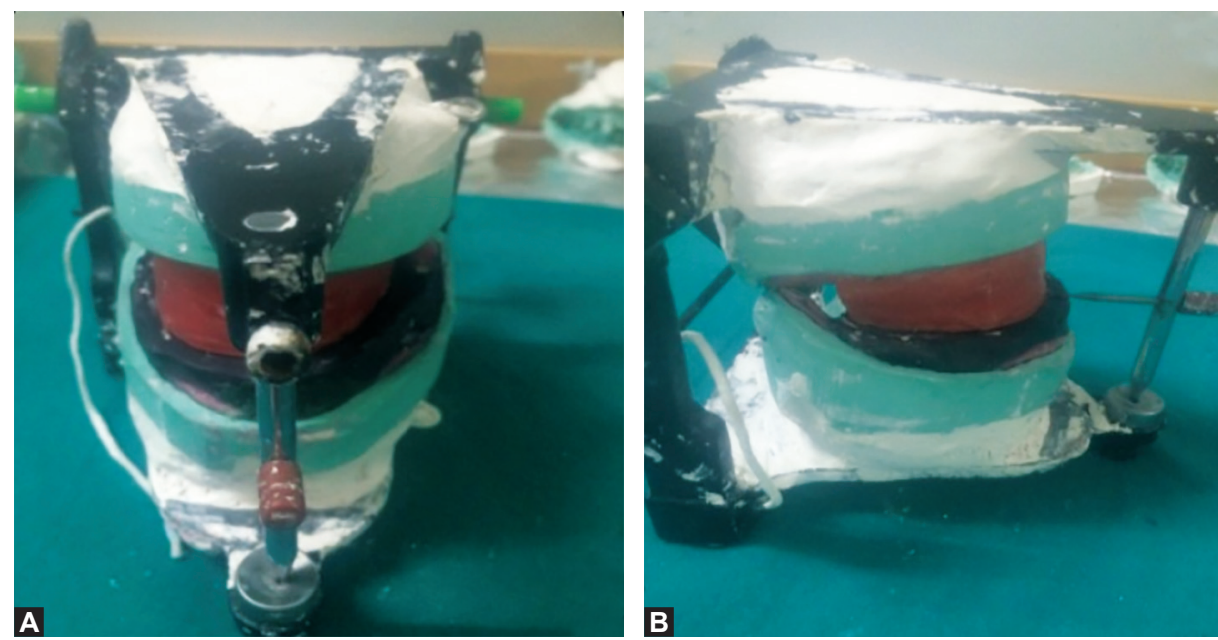

Figs 4A and B: Record rims after functional molding

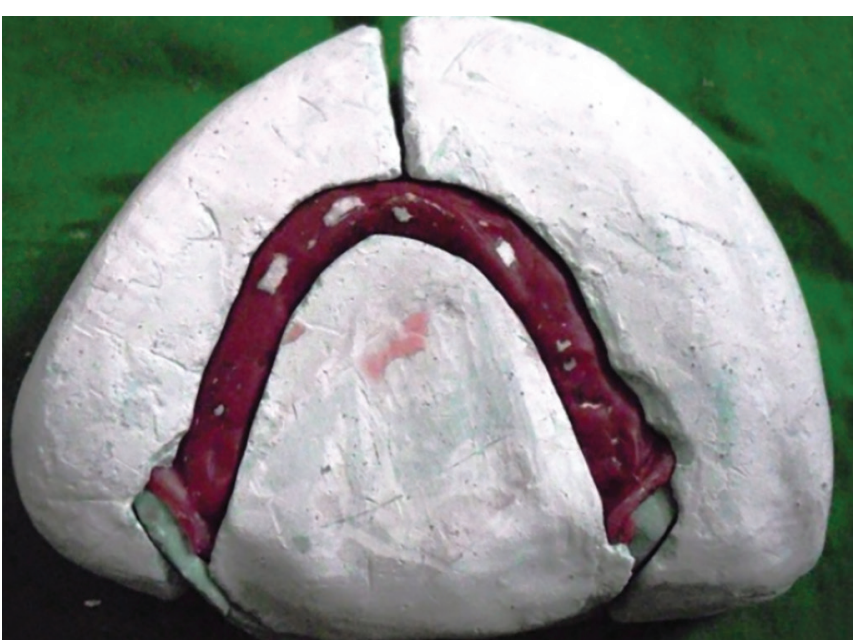

Fig. 5: Plaster indices around molded impression compound

pout/purse lips, count from 60 to 70, talk aloud, pronounce the vowels, sip water, swallow, slightly protrude the tongue and lick the lips. These actions were repeated for 10 minutes until the compound became hard.

- Then the maxillary rim was adjusted at the same vertical height with mandibular rim maintaining the vertical stop. Same actions were repeated again for 10 minutes.

- Both the compound rims were then replaced on the articulator to evaluate the vertical relation again. (Figs 4A and B).

\section{Laboratory Stages}

- The compound rims were placed on the duplicate master cast and the locating grooves were cut. The plaster index was made around the molded impression compound rim (Fig. 5).

- Then the molded impression compound rims were removed from the base plate and the index was

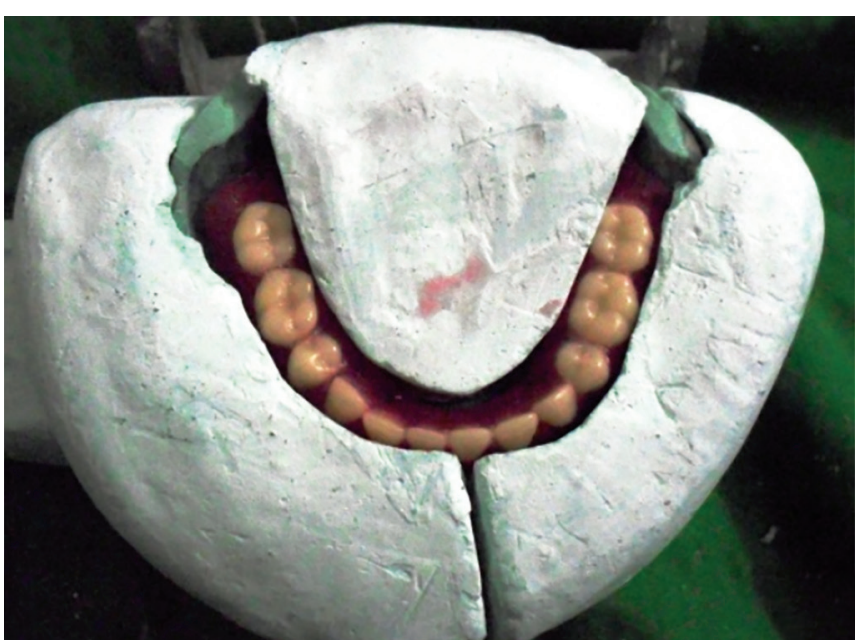

Fig. 6: Teeth arrangement according to plaster indice

replaced. The index preserve the space of the neutral zone.

- Wax was then poured into the space giving an exact representation of the neutral zone.

- The newly formed wax rims were then replaced on the articulator.

- Teeth arrangement was done exactly following the indices. During the setting up of the teeth their position was checked by putting the indices together around the wax try-in (Fig. 6).

- Modification of the artificial posterior teeth had to be done to accommodate it on the narrow space of neutral zone.

- Wax try-in was done.

- Dentures were processed and finished in a routine manner. Polishing was done lightly so as to preserve the contour of the flanges.

- Dentures were finally inspected and clinical remounting was done to eliminate minor occlusal errors.

- Insertion of finished maxillary and mandibular dentures were done (Fig. 7). 


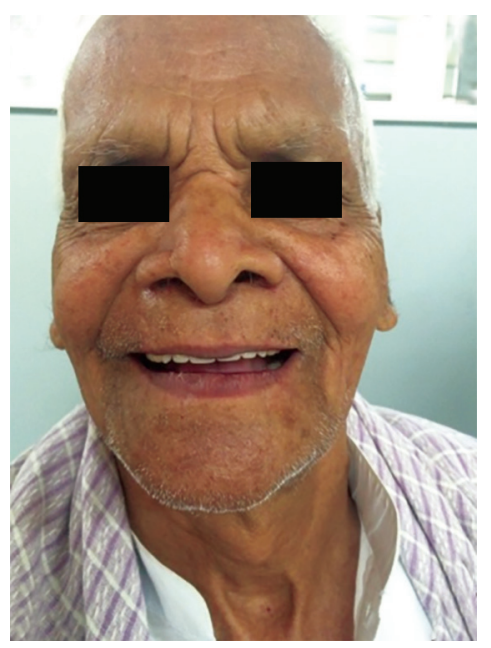

Fig. 7: Finished complete intraoral dentures

\section{DISCUSSION}

The ultimate aim of Prosthodontics is to restore form, function and esthetics. Pound ${ }^{14}$ pointed out that in advanced resorption, the maxillary ridge moves lingually and mandibular ridge moves buccally. Atwood ${ }^{9}$ divides the factors related to the rate of resorption into the categories of anatomic, metabolic, functional, and prosthetic. Our knowledge of these factors is imperfect, we know that resorption may take place despite our best efforts at prevention. The neutral zone approach registers the neutral zone to determine the proper placement of teeth after resorption has taken place. It is the opinion of many that the maxillary anterior teeth should be placed close to the position of the natural anterior teeth. If this is not accomplished, esthetics and phonetics might be compromised. In addition, the position, of the maxillary teeth must satisfy the desires of the patient. Neutral zone registration may dictate that the mandibular anterior teeth be placed more lingually. This usually can be accomplished without sacrificing esthetics. When the patient functionally molds the maxillary and mandibular rim into the area of the neutral zone, the result is a more stable denture.

\section{CONCLUSION}

The use of the neutral zone concept for denture fabrication has been advocated since the 1930s. The neutral zone is an alternative technique for the construction of complete dentures on highly atrophic ridges. It is especially useful in cases where dental implants are not possible. The aim of the neutral zone is to construct a denture in muscle balance, as muscular control will be the main stabilizing and retentive factor during function. The technique is relatively simple but there are increased chair time and laboratory costs to be considered.

\section{REFERENCES}

1. Weinberg LA. Tooth position in relation to the denture base foundation. J Prosthet Dent 1958;8:398-405.

2. Bolender CL, Smith DE. Diagnosis and management of inadequate denture prostheses. In: Laney WR, Gibilisco JA, editors. Diagnosis and treatment in prosthodontics. Philadelphia, PA, Lea and Febiger 1983:279-282.

3. Wright CR. Evaluation of the factors necessary to develop stability in mandibular dentures. J Prosthet Dent 2004;92:509518.

4. Fahmy FM, Kharat DU. A study of the importance of the neutral zone in complete dentures. J Prosthet Dent 1990;64:459-462.

5. Beresin VE, Schiesser FJ. The neutral zone in complete dentures. J Prosthet Dent 2006;95:93-100.

6. Schiesser FJ. The neutral zone and polished surfaces in complete dentures. J Prosthet Dent 1964;14:854-865.

7. Raybin NH. The polished surface of complete dentures. J Prosthet Dent 1963;13:236-239.

8. Basker RM, Harrison A, Ralph JP. A survey of patients referred to restorative dentistry clinics. Br Dent J 1988;164:105-108.

9. Atwood DA. Postextraction changes in the adult mandible as illustrated by micrographs of midsagittal sections and serial cephalometric roentgenograms. J Prosthet Dent 1963;13:810-824.

10. Ohukubo C, Hantani S, Hosoi T, Mizuno Y. Neutral zone approach for denture fabrication for a partial glossectomy patient: a clinical report. J Prosthet Dent 2000;84:390-393.

11. Fish EW. An analysis of the stabilising factors in full denture construction. Br Dent J 1931;52:559-570.

12. The glossary of prosthodontic terms. J Prosthet Dent 2005;94:10-92.

13. Sheppard IM. Denture base dislodgment during mastication. J Prosthet Dent 1963;13:462-468.

14. Pound E. Lost-Fine arts in the fallacy of the ridges. J Prosthet Dent 1954;4:6-16. 